\title{
Tchekov em Portugal
}

\author{
Rui Pina Coelho
}

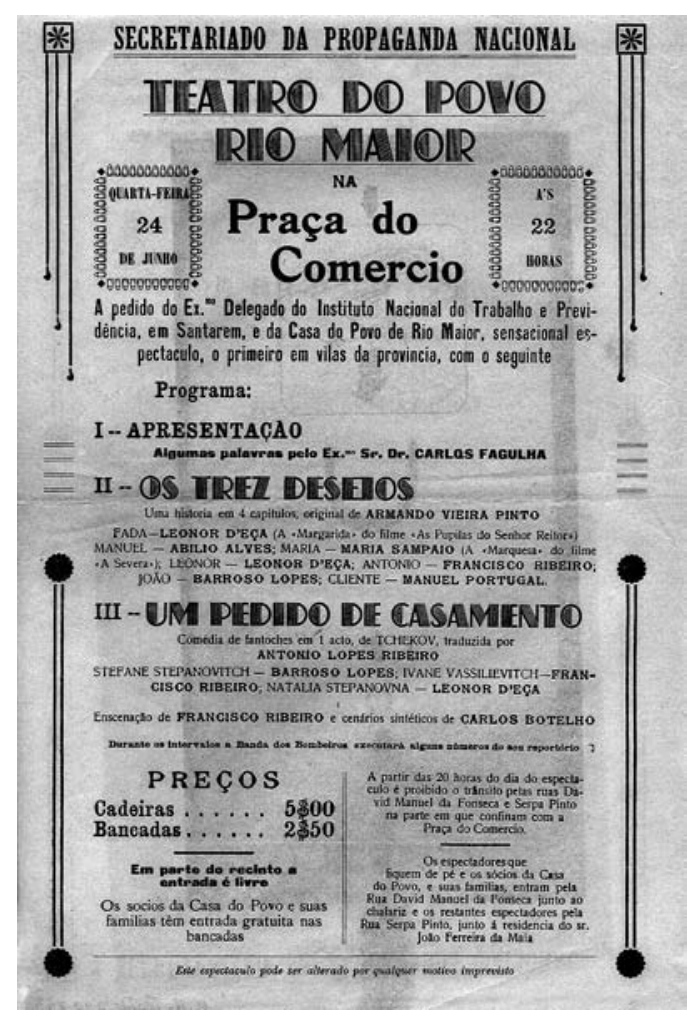

\section{Uma presença estruturante}

Anton Pavlovich Tchekov (1860-1904) chega a Portugal primeiro às páginas literárias que aos palcos. Em 1909, cinco anos depois da consagração na mítica estreia d' $A$ gaivota no Teatro de Arte de Moscovo, para Eduardo de Noronha, em Evolução do teatro: 0 drama através dos séculos, Tchekov não passa de mais um dramaturgo entre uma plêiade de autores russos que terão seguido as passadas de Pushkin (1909: 351-352). Armando Ferreira, em 1923, lamenta que até então o autor russo não tenha sido representado entre nós (1923: 5-6). Mais tarde, em 1924, Augusto de Lacerda, na obra Teatro futuro: Visão de uma nova dramaturgia, declara sumariamente que o autor "não teve discípulos que o honrassem" (1924: 29). Um ano depois, António Ferro, numa entrevista ao Diário de Lisboa (18-2-1925), enumerava o autor russo entre as novidades da dramaturgia europeia que o seu Teatro Novo tencionava levar à cena - contudo, dada a efemeridade do projecto, este intuito acabaria por sair gorado.

Um tratamento mais aprofundado a Tchekov é feito por Eduardo Scarlatti, em 1927, na obra Ideias de outros, onde afirma que "as peças de teatro de Tchekov significam mais uma aspiração de movimento cénico para as figuras dos seus contos do que a realização teatral de efeitos

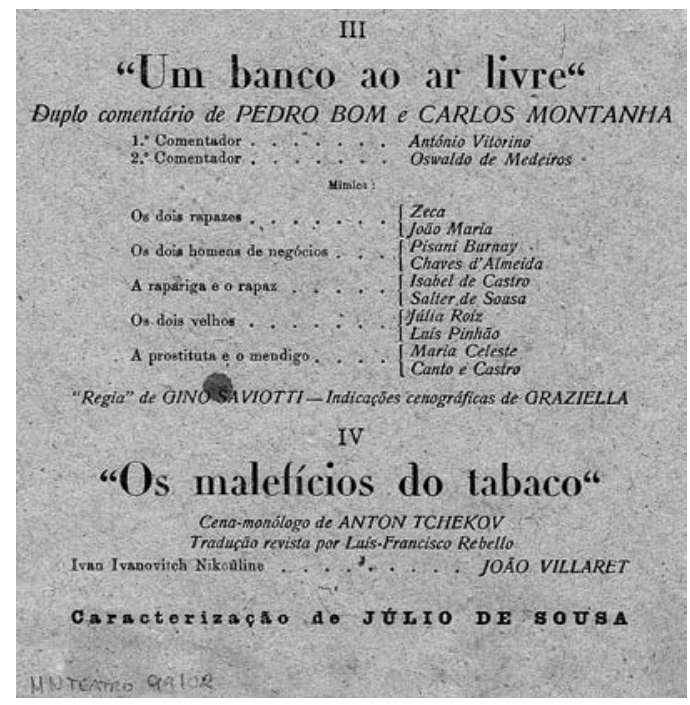

\section{Folheto de apresentação dos espectáculos \\ Os trez desejos, de \\ Armando Vieira Pinto, e Um pedido de casamento em Rio Maior, enc. Francisco Ribeiro, Teatro do Povo, 1936 (cortesia do Museu} Nacional do Teatro).

Programa do $5^{\circ}$ espectáculo essencialista londe se inclui Os maleficios do tabaco), Teatro-Estúdio do Salitre, 1947 (cortesia do Museu Nacional do Teatro). preconcebidos" (1927: 64). Em 1934, é a vez de Augusto Ricardo, em Motivos de teatro, notar que, para a renovação da arte dramática, Stanislavski "serviu-se, principalmente, do teatro psicológico de Antão Chekow [sic]" (1934: 84).

Aos palcos só chegará em 1936 pela mão de Francisco Ribeiro com o Teatro do Povo, numa encenação de Um pedido de casamento, com interpretações de Barroso Lopes, do próprio Ribeirinho e de Leonor d'Eça. A tardia aproximação do teatro português a Tchekov parecia fazer eco das palavras de Scarlatti, quando afirmava: "não há na Europa Ocidental intérpretes para tais figuras, como não há público para as sentir e compreender" (1927: 66).

Entendido assim como um autor difícil e com relações pouco favoráveis com a censura, a estreia de uma "peça longa" só acontecerá na segunda metade do século XX, com o Teatro d'Arte de Lisboa, de Orlando Vitorino e Azinhal Abelho, numa encenação de As três irmãs (1955), onde é sobretudo valorizada a intenção de fazer um "teatro sério em que a distracção é substituída pela concentração e pelo esforço mental" (Diário de Lisboa, 4-1-1956). Até esta data, a presença de Tchekov é pautada pela apresentação das peças em um acto, que irão ter sempre uma presença assídua nos palcos portugueses, especialmente entre o teatro de amadores, o que para 
Os maleficios do tabaco, enc. António Pedro,

TEP - Teatro Experimental do Porto, 1955 (Jaime Valverde), (cortesia do Teatro Experimental do Porto).

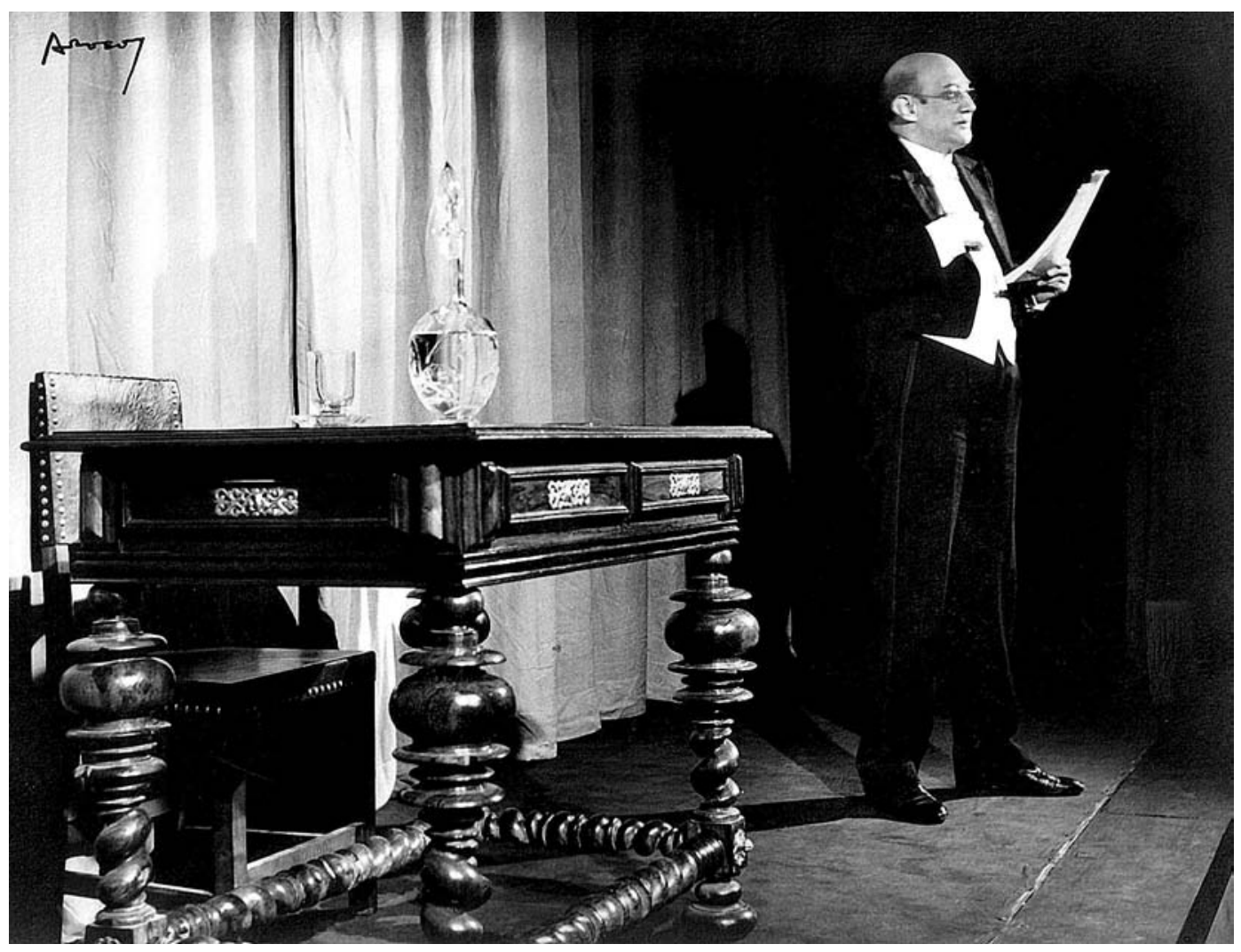

muito terá contribuido a publicação, no início dos anos sessenta, de Seis peças em um acto (Tchekov 1960) e Duas peças em um acto (Tchekov 1961).

Durante a segunda metade dos anos quarenta, momento em que a cena portuguesa ensaia uma constantemente adiada renovação cénica, Tchekov vai ver-se associado aos grupos que preconizam esta vontade de mudança. Assim, o autor russo vai integrar os repertórios do TeatroEstúdio do Salitre, dos Companheiros do Pátio das Comédias, do Grupo Dramático Lisbonense, do Teatro Experimental do Porto (TEP) e do já referido Teatro d'Arte de Lisboa. Idêntica associação ocorrerá com aqueles colectivos que, num segundo fôlego de renovação, vão dar seguimento à gorada revitalização da cena portuguesa, tais como o Teatro dos Estudantes da Universidade de Coimbra (TEUC), o Teatro d'Ensaio, o Teatro-Estúdio de Lisboa (TEL) e, até mesmo o grupo de amadores da Sociedade Guilherme Cossoul.

É neste contexto que as peças longas vão ser estreadas entre nós. Depois de As três irmãs de 1955, é a vez de 0 tio Vânia, pelo TEP, uma encenação de João Guedes, num "espectáculo cheio de dignidade, notável entre nós" (Porto 1973: 82). Em 1965, Luzia Maria Martins, pelo TEL, estreia 0 pomar das cerejeiras, numa versão cenicamente depurada, entendida como uma iniciativa "que teima em querer dar a Lisboa o teatro que Lisboa não merece" (Manuela de

do texto de Joaquim Pacheco Neves (19101998), Anton Tchekov: Drama em 3 actos, Vila do Conde, Edições Ser, 1988 Recebeu o $3^{\circ}$ prémio no concurso de originais do Ciclo de Teatro de Autores Portugueses (CITAP), em 1987 e, ao que sabemos, nunca foi representado.
Academia de Santo Amaro, em 1965, numa encenação de Alexandre Babo.

A última estreia de uma peça longa dá-se no início de 1974, numa encenação de Platonov por Jorge Listopad para a Companhia de Teatro da RTP. Embora a esta encenação se tenham reconhecido algumas debilidades, não deixou de ser saudada como um espectáculo "a vários títulos respeitável e digno" (Maria Helena Dá Mesquita, A capital, 12-1-1974).

A presença de Anton Tchekov em Portugal generaliza-se e, tornando-se um consensual autor de repertório, ganha também novas dimensões. Assim, aparece nos palcos portugueses pela mão de companhias estrangeiras que aportam novas propostas cénicas, de The Wooster Group (Brace Up!, 1992) ao Teatro Krétakör (Siráj, 2005), passando por Nekrosius (Trys Seserys, 1997). A obra ou a própria figura de Tchekov constituem-se também como matéria de revisitação dramática', como acontece em Tchekov-

Tchekova, de François Nocher (1986), Caixa preta, de Lúcia Sigalho (2002 e 2003) ou Ensaio, de José Peixoto (2003), podendo ser ainda citados os casos de Tio Vânia, de Howard Barker (2000), ou Uma peça mais tarde e 0 jogo de lalta, de Brian Friel (2003). 0 fascinio pelo universo tchekoviano não se queda pela encenação dos seus textos, nem pela (re)escrita dramática, contagiando mesmo outras artes performativas como a dança - em Silence So Sexy (Francisco Camacho, 2002) e A última dança (Elisa Worm, 1998) -, ou a ópera, com The Bear, de William Walton (1974 e 1990).

Não deixa de ser curioso que, sendo Anton Tchekov um dos autores de eleição para um teatro de repertório, se veja arredado dos palcos do Teatro Nacional desde 1980, e só timidamente apareça nas programações das companhias mais consagradas. Curioso é também reparar que, nos últimos anos, são grupos de carácter mais experimental (ou alternativo?) que se aventuram nos 


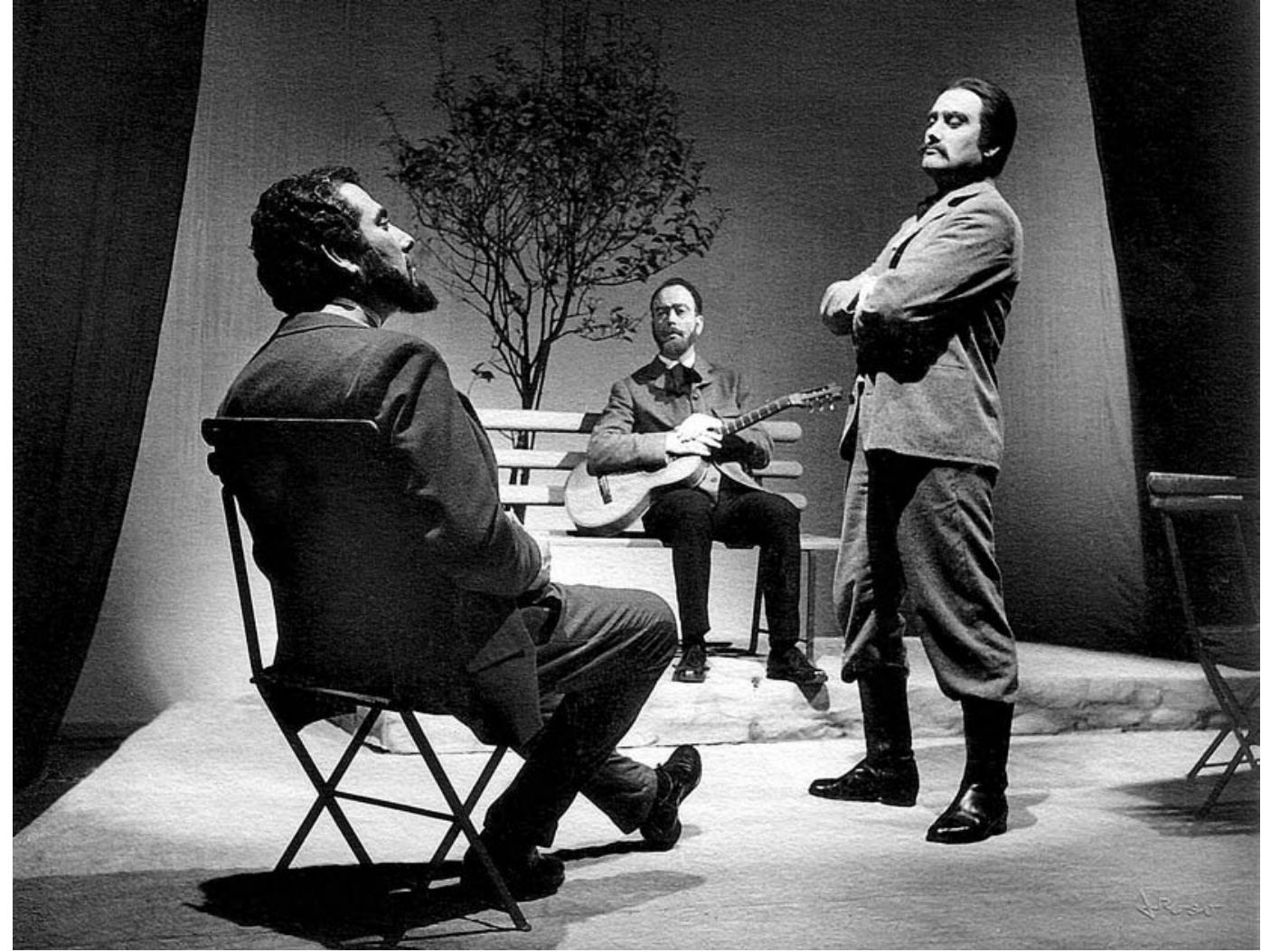

dominios tchekovianos, tais como o Cão Solteiro, a Sensurround ou a Casa Conveniente. Por tudo isto, cremos que será difícil encontrar um autor que acompanhe tão marcadamente a evolução do teatro em Portugal como este médico russo, Anton Tchekov, sobre cujo desaparecimento passaram, em 2004, cem anos, oportuna razão para esta modesta homenagem da Sinais de cena à sua presença nos palcos portugueses.

\section{Lista de espectáculos encenados}

1936. Um pedido de casamento. Trad. António Lopes Ribeiro. Enc. Francisco Ribeiro. Prod. Teatro do Povo.

1941. $O$ urso. Dir. Alfredo Ruas. Prod. Teatro do Povo.

1945. $O$ urso. Enc. António Lopes Ribeiro. Prod. Os Comediantes de Lisboa.

1947. Os malefícios do tabaco. Trad. revista por Luiz Francisco Rebello. Prod. Teatro-Estúdio do Salitre.

1948. $O$ canto do cisne. Trad. Avelino Cunhal. Enc. Costa Ferreira. Prod. Os Companheiros do Pátio das Comédias.

1949. O trágico à força. Enc. Manuela Porto. Prod. Grupo Dramático Lisbonense.

O urso. Trad. Luiz Francisco Rebello. Enc. Manuela Porto. Prod. Grupo Dramático Lisbonense. 0 aniversário do banco. Trad. Augusto Casimiro. Enc. Manuela Porto. Prod. Grupo Dramático Lisbonense.

1953. Um pedido de casamento. Trad. Correia Alves. Enc. António Pedro. Prod. Teatro Experimental do Porto (TEP).

1955. Exercícios de teatro: Malefícios do tabaco. Trad. Luiz Francisco Rebello. 0 canto do cisne. Trad. Pedro Serôdio. $O$ trágico à força. Trad. Correia Alves. Enc. António Pedro. Prod. TEP.
As três irmãs. Trad. Azinhal Abelho e Orlando Vitorino. Enc. Orlando Vitorino e Azinhal Abelho. Prod. Teatro d'Arte de Lisboa.

1957. Tchekov: $O$ urso. Trad. Francisco Ribeiro. $O$ canto do cisne. Trad. Pedro Serôdio. Os malefícios do tabaco. Trad. Luiz Francisco Rebello. Um pedido de casamento. Trad. António Pinheiro. Dir. João Sarabando e Alexandre Passos. Prod. Teatro d'Ensaio. Tchekov, Molière, Raul Brandão: Malefícios do tabaco. Trad. Luiz Francisco Rebello. Dir. João Sarabando. Prod. Teatro d'Ensaio.

Um pedido de casamento. Prod. Sociedade de Instrução Guilherme Cossoul.

1958. $O$ urso. Prod. Conjunto Cénico Caldense.

Rebello. Enc. Carlos Garrido. Prod. Conjunto Cénico Caldense.

Tchekov, Pirandello, Salacrou: 0 canto do cisne. Trad. Pedro Serôdio. Enc. Alexandre Passos. Prod. Teatro d'Ensaio.

1959. $O$ urso. Prod. Círculo Experimental de Teatro de Aveiro (CETA).

1960. ${ }^{3} O$ tio Vânia. Trad. Armando Bacelar. Enc. João Guedes. Prod. TEP.

O urso. Enc. Paulo Quintela. Prod. Teatro dos Estudantes da Universidade de Coimbra (TEUC). O urso / Os malefícios do tabaco / Um pedido de casamento. Enc. Mário Temido. Prod. Secção de Teatro do Ateneu de Coimbra.

1962. La mouette. Enc. Sacha Pitoëff. Os malefícios do tabaco. Trad. Luiz Francisco Rebello. Prod. TEP.

1965. O pomar das cerejeiras. Trad. Luzia Maria Martins. Enc. Luzia Maria Martins. Prod. Teatro Estúdio de Lisboa (TEL).
Os malefícios do tabaco. Trad. Luiz Francisco
O tio Vânia,

enc. João Guedes, TEP - Teatro Experimental do Porto, 1960 (João Guedes, José Silva e Baptista Fernandes), (cortesia do Teatro Experimental do Porto).

Esta lista integra

montagens, adaptações ou reescritas, bem como encenações de textos

originais a partir da vida e obra de Tchekov. Nestes casos, os respectivos titulos são registados entre parênteses após 0 título do espectáculo

sendo o seu autor devidamente identificado. Nos casos em que o espectáculo contém textos de diferentes autores regista-se somente a informação relativa a Tchekov. Para informações mais detalhadas sobre

qualquer um destes espectáculos consulte-se a CETbase:

http://www.fl.ul.pt/centroestudos-teatro.htm.

3 Centenário do nascimento de Anton Tchekov. 


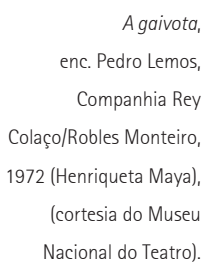

Confissões numa esplanada de Verão

(onde se inclui

Trágico à força), enc. Mário Viegas, 1984

(Carmen Dolores,

Melim Teixeira e

Carlos Pisco),

(cortesia do Museu

Nacional do Teatro).
A gaivota. Enc. Alexandre Babo. Prod. Teatro da Academia de Santo Amaro.

O urso / Aniversário do banco. Enc. Abílio Mendes. Prod. Grupo Cénico dos Estudantes de Medicina de Lisboa.

Pedido de casamento. Prod. CETA.

Malefícios do tabaco. Prod. CETA.

1967. O urso. Prod. Grupo da Associação de Estudantes do Instituto Superior Técnico.

Malefícios do tabaco. Enc. colectiva. Prod. Grupo Cénico do Grupo Phillips.

1968. O pedido de casamento. Enc. Virgílio Macieira. Prod. Grupo de Teatro Amador da Santa Casa da Misericórdia.

Malefícios do tabaco. Prod. CETA.

Um pedido de casamento/ $O$ aniversário do banco. Prod. Teatro de Ensaio do Barreiro.

1970. Um pedido de casamento. Enc. José de Almeida. Prod. Grupo Cénico da Delegação da FNAT de Coimbra.

1972. A gaivota. Trad. Natércia Freire (sobre texto de Elsa Triolet). Enc. Pedro Lemos. Prod. Companhia Amélia Rey Colaço / Robles Monteiro.

Trilogia: $O$ urso, Aniversário do banco e Um pedido de casamento. Enc. Marcelo Brito. Prod. Grupo de Intervenção Teatral da Trafaria (GIT).

1974. The Bear (The Bear, 1967). Autor do libreto: William Walton. Prod. companhia estrangeira não identificada [ópera].

Um pedido de casamento / 0 aniversário do banco / $O$ urso. Prod. Grupo Cénico Paroquial de Arroios.

Platonov. Trad. Natália Correia. Enc. Jorge Listopad. Prod. Companhia RTP / Teatro Municipal Maria Matos.

1975. $O$ urso. Enc. Ruy de Matos. Prod. Grupo de Teatro e Música dos Trabalhadores da Manutenção Militar.

1977. As três irmãs. Enc. Rogério de Carvalho. Prod. GITT. Variações nostálgicas [a partir de Três irmãs]. Coreografia: Armando Jorge. Prod. Ballet Gulbenkian [dança].

1980. As três irmãs. Dramaturgia: Fernanda Alves. Enc Costa Ferreira. Prod. Teatro Nacional D. Maria II. Tio Vânia. Enc. Rogério de Carvalho. Prod. Teatro na Caixa.

1981. O urso / Um pedido de casamento. Prod. A Capoeira de Barcelos.

Jornada teatral [integra excertos de A gaivota]. Enc. Adolfo Gutkin. Prod. Teatro Maizum.

1982. A gaivota. Trad. Teatro do Mundo (revista por Emília Amor). Enc. Rogério de Carvalho. Prod. Teatro do Mundo.

0 urso. Prod. Gente Nova.

1983. Textos de Tchekov. Enc. Fernando Lobo. Prod. Grupo Praça Pública.

Malefícios do tabaco. Prod. Grupo de Teatro de Barroselas.
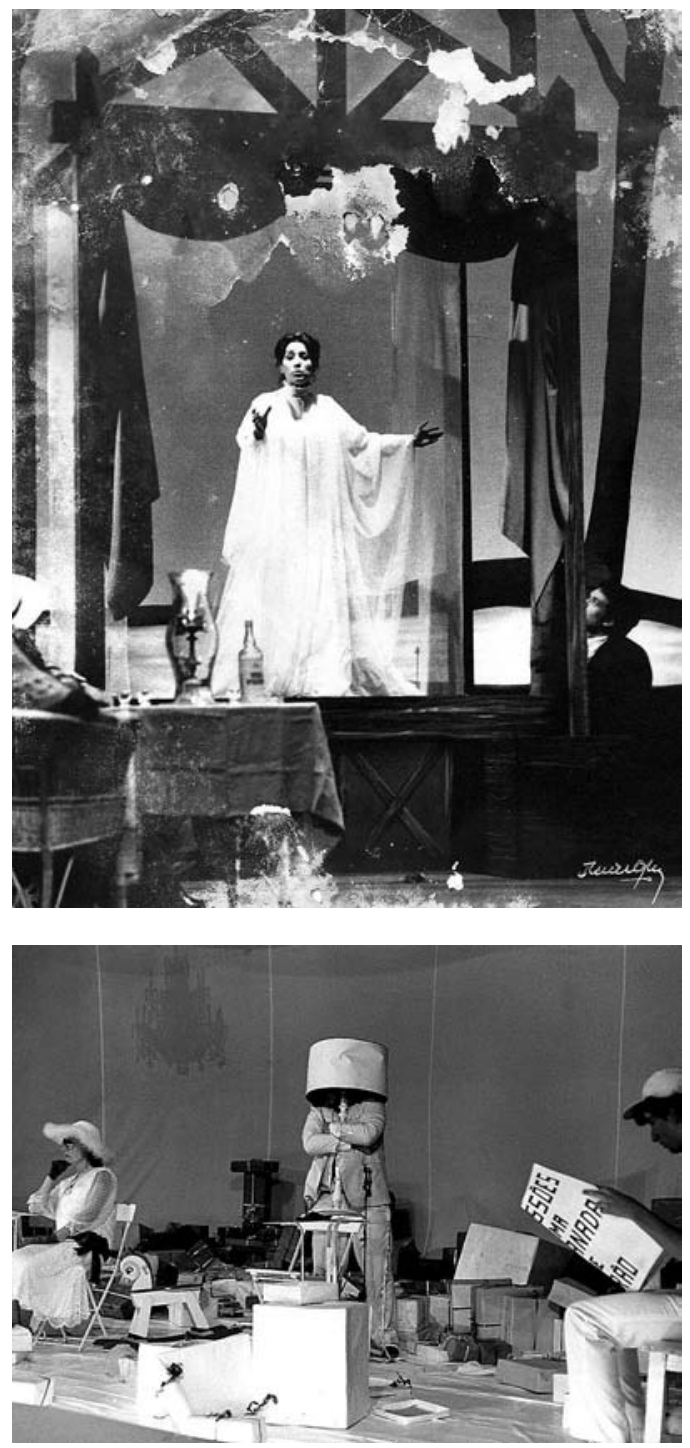

1984. O canto do cisne / Malefícios do tabaco. Enc. Alexandre Passos e José Caldeira. Prod. Centro Cultural de Évora.

Confissões numa esplanada de Verão: 0 trágico à força. Trad. Luiz Francisco Rebello. Dir. Mário Viegas. Prod. Novo Grupo/ Teatro Aberto.

0 urso. Enc. Alexandre Parracho. Prod. Presença Grupo de Teatro de Amadores de Setúbal.

1985. Os malefícios do tabaco. Enc. José Gonçalinho. Prod. Teatro Art'Imagem.

Malefícios do tabaco / 0 urso. Enc. Silvina Pereira. Prod. Grupo de Teatro da Casa do Pessoal da Santa Casa da Misericórdia de Lisboa.

Ai que saltos me dá o coração: Um pedido de casamento. Versão portuguesa: Teatro Ibérico. 0 urso. Trad. Luiz Francisco Rebello. 0 aniversário no banco. Versão portuguesa: Teatro Ibérico. Enc. Xosé Blanco Gil. Prod. Teatro Ibérico.

Um pedido de casamento. Enc. João de Melo Alvim. Prod. Teatro da Sociedade - Grupo de Teatro da Sociedade União Sintrense.

1986. Tchekov, Tchekova (Tchékhov-Tchékhova, 1986). Autor: François Nocher. Enc. François Nocher. Prod. Instituto Franco-Português.

O urso. Enc. Ventura Rodrigues. Prod. Água Viva. 

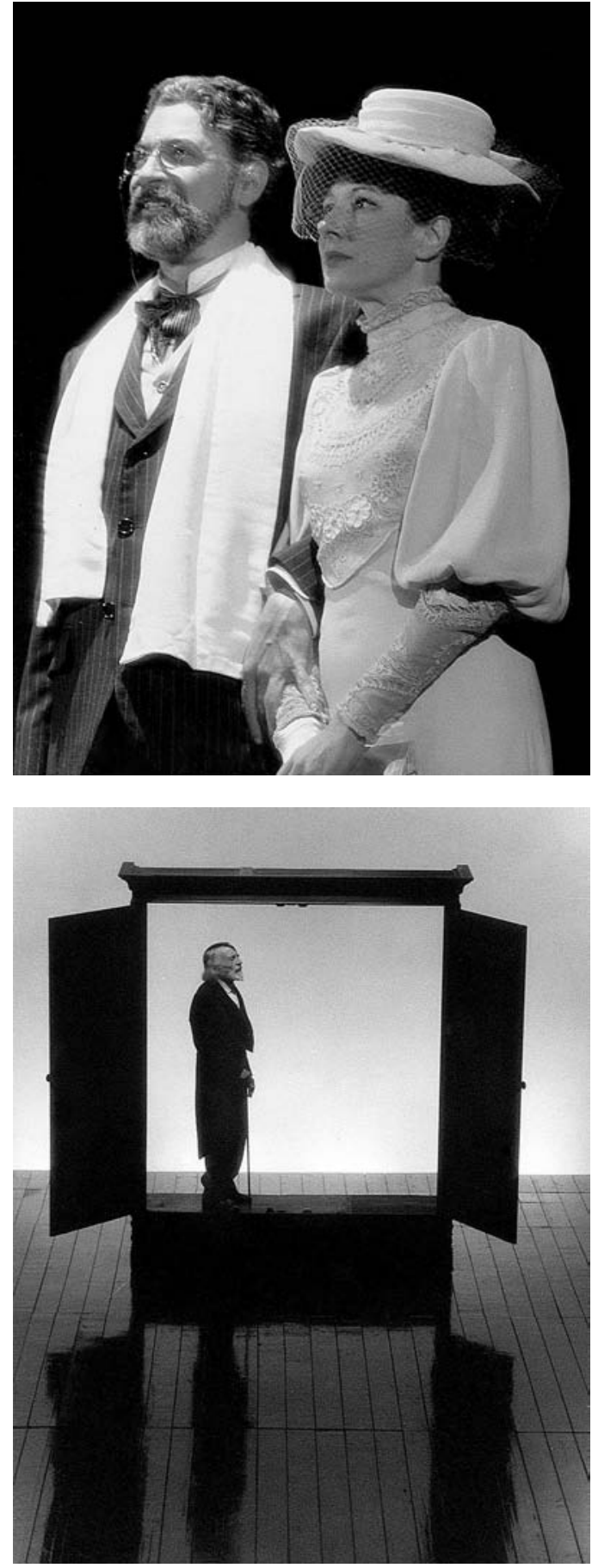

Um pedido de casamento. Enc. David Carvalho. Prod. Filandorra - Teatro do Nordeste / Centro Cultural Regional de Vila Real.

Suspiros: 0 urso. Enc. António Durães. Prod. Grupo Cénico da Associação Naval $1^{\circ}$ de Maio.

1987. Tio Vânia. Trad. Armando Bacelar. Enc. Fernando Umaña. Prod. Seiva Trupe.

0 jardim das cerejas. Versão: João Lourenço e Vera San Payo de Lemos. Enc. João Lourenço. Prod. Novo Grupo / Teatro Aberto.

Pedido de casamento / 0 urso. Enc. Cucha Carvalheiro. Prod. Grupo de Teatro Amador do Instituto Nacional de Meteorologia e Geofisica "Boas Abertas".

1988. Três irmãs. Trad. Augusto Sobral, Carol Loff, Rui
Mendes. Enc. Rui Mendes. Prod. Teatro da Cornucópia.

Deus os fez, Deus os juntou: 0 nome do cavalo Pedido de namoro, A corista, Uma natureza enigmática [contos], 0 canto do cisnee $A$ gaivota. Adaptação: Mário Viegas e Manuela de Freitas. Enc. Mário Viegas. Prod. Mário Viegas e Manuela de Freitas.

Os malefícios do tabaco. Trad. Luiz Francisco Rebello. Prod. Teatro d' Ensaio.

0 urso. Enc. Carlos Santos. Prod. Grupo Paroquial de Teatro de Leça da Palmeira.

1989. $O$ filho do ar: $O$ canto do cisne. Trad. Manuel João Gomes. Enc. Carlos Fernando. Prod. Grupo Teatro Hoje.

Jardim das cerejeiras. Trad. Carlos Porto (revista por Gueorgui Kristovsky). Enc. Rogério de Carvalho. Prod. Teatro Estúdio de Arte Realista (TEAR).

$O$ urso. Enc. Rui Sena. Prod. Grupo de Intervenção Cultural da Covilhã (GICC).

1990. Roleta Russa: $O$ urso, Os malefícios do tabaco, $O$ pedido de casamento e No comboio [conto]. Prod. Teatro Animação de Setúbal (TAS).

Platonov. Trad. Carlos Porto. Enc. Rogério de Carvalho. Prod. TEUC.

Façade / 0 urso (The Bear, 1967). Autor do libreto: William Walton. Enc. Luís Miguel Cintra. Prod. Teatro da Cornucópia e RTP [ópera].

O cerejal. Enc. Otomar Krejca. Prod. Za Branou II. As três irmãs. Enc. Vague Sakheveerdian. Prod. Teatro Kirovokan

A última palavra? Enc. João de Melo Alvim. Prod. Teatro da Sociedade - Grupo de Teatro da Sociedade União Sintrense.

Tchekhov e o limão espremido: 0 urso, Um pedido de casamento e $\boldsymbol{O}$ canto do cisne. Enc Rui Pinto. Prod. GITT.

Um pedido de casamento. Enc. Manuel Ângelo. Prod. Grupo Dramático Beneficente de Vilar do Paraiso.

0 urso. Prod. Teatro de Papel.

Pressentimentos... amores... de Tchekov Biografia teatralizada de Tchekov: Os malefícios do tabaco, $O$ canto do cisne, Um pedido de casamento e A gaivota [excerto]. Enc. Armando Caldas. Prod. Intervalo - Grupo de Teatro.

1991. As suaves alegrias da felicidade conjugal: Os malefícios do tabaco. Trad. Rui Mendes. 0 urso. Trad. Luiz Francisco Rebello. Um pedido de casamento. Trad. Rui Mendes. Enc. Rui Mendes. Prod. Teatro da Malaposta / Centro Dramático Intermunicipal Almeida Garrett (CDIAG)

Tio Vânia. Enc. Jo Van Osselt. Prod. Nova Comédia Bracarense.

1992. A gaivota. Trad. Fiama Hasse Pais Brandão. Enc. Gastão Cruz. Prod. Grupo Teatro Hoje.

\section{Tchekov-Tchekova, de François Nocher, enc. François Nocher Instituto Franco- Português, 1986 (Mário Jacques e Fernanda Lapa),} (cortesia do Museu Nacional do Teatro).

O jardim das cerejas enc. João Lourenço, Novo Grupo, 1987 (Luis Santos) cortesia do Museu Nacional do Teatro). 

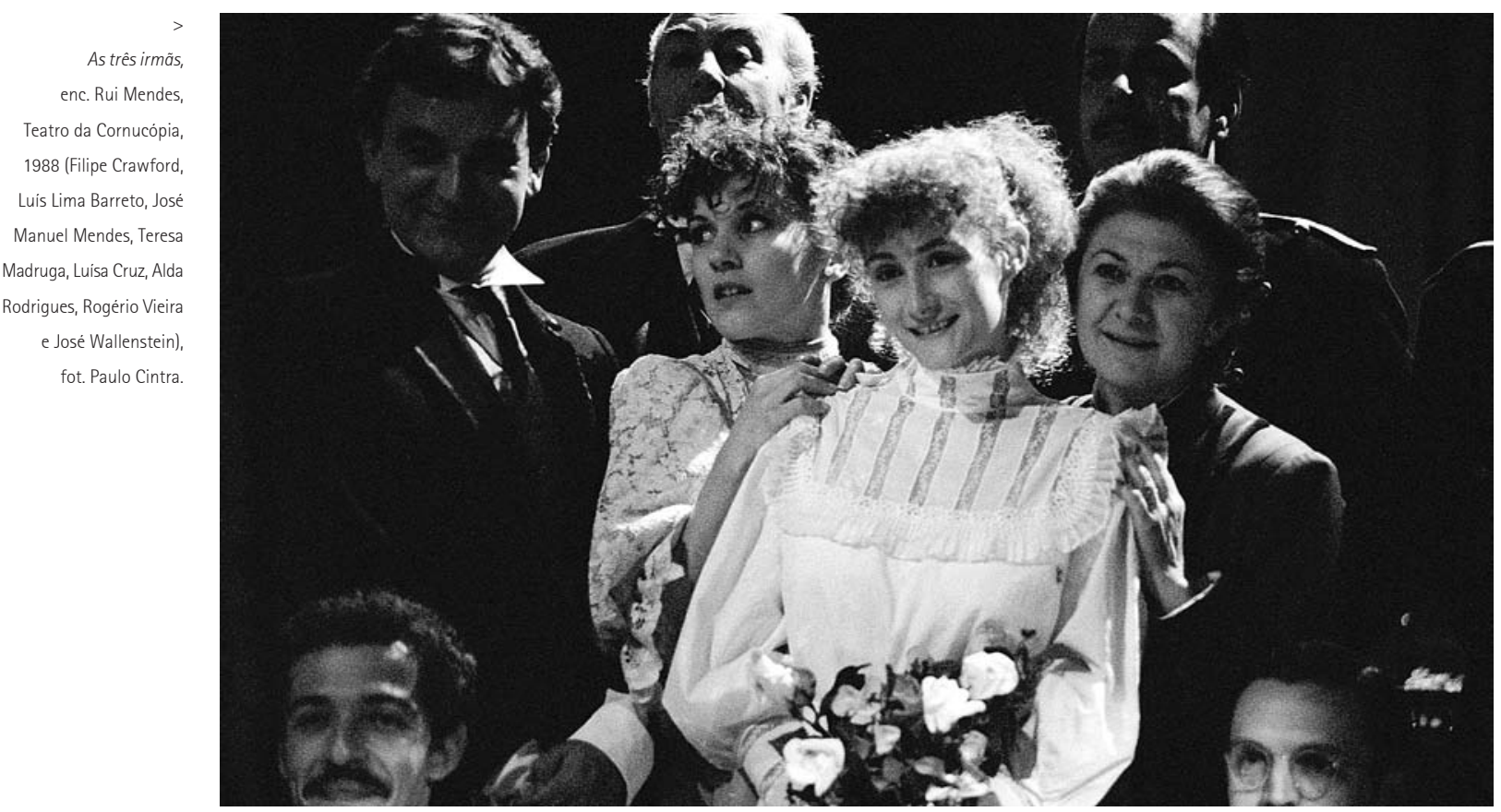

Brace Up! [a partir de Três irmãs]. Trad. (para inglês) Paul Schmidt. Enc. Elizabeth LeCompte. Prod. The Wooster Group.

Concerto em um acto para dois actores e duas marionetas. Enc. José Ramalho.

Pedido de casamento. Prod. Teatro da Meia Lua.

1993. Um pedido de casamento. Trad. Correia Alves. Enc. José Cayolla. Prod. TEP.

$O$ urso / Um pedido de casamento. Enc. João Bilou. Prod. Sociedade Operária de Instrução e Recreio Joaquim António d'Aguiar (SOIR).

1994. Três passagens para Moscovo [a partir de Três irmãs]. Adap. Luísa Costa Gomes. Enc. Polina Klimotivska. Prod. Centro Cultural de Belém.

1995. Ursos e cisnes. Enc. José Mascarenhas e Victor Pires. Prod. Teatro de Portalegre.

Pedido de casamento. Enc. Isabel Pais. Prod. Grupo de Teatro Amador Grão-de-Bico.

Os prados: Um pedido de casamento, Malefícios do tabaco e 0 urso. Enc. colectiva. Prod. Eléctrico. 0 copo d'água. Enc. Rodolfo Garcia Vasquez. Prod. Os Silenos.

De Moscovo a Lisboa. Enc. Filipe Marinho Bastos. Prod. Grupo de Teatro "Os Jotas".

0 urso. Enc. João de Melo Alvim. Prod. Companhia de Teatro de Sintra.

1997. Coração, coração: $O$ urso e Um pedido de casamento. Enc. João de Melo Alvim. Prod. Companhia Teatro de Sintra - Chão de Oliva. Trys Seserys [Três irmãs]: Enc. Eimuntas Nekrosius. Prod. Meno Fortas.

Platonov. Enc. Andrzej Sadowoski. Prod. Escola Superior de Música e Artes do Espectáculo (ESMAE) / Teatro Nacional S. João.

1998. Três irmãs. Trad. Natália Luiza. Enc. Natália Luiza. Prod. Novo Núcleo de Teatro da F.C.T. da Universidade Nova de Lisboa (NNT). Tio Vânia. Trad. Rui Mendes. Enc. Rui Mendes. Prod. Teatro da Malaposta / CDIAG. Point Blank [Platonov]. Trad. Michael Frayn.
Espectáculo de: Adriaan van den Hoof, An d'Huys, Cristina Bizarro, Dinarte Branco, Eefke Saris, Frank Vercruyssen, Gillis Bisheuvel, Günter Lesage, Inne Box, Jolente de Keersmaeker, Luz da Câmara, Pedro Penim, Renild van Bavel, Sara de Roo, Thomas Walgrave, Tiago Rodrigues, Tine Embrechts. Prod. Tg Stan.

A felicidade conjugal: Um pedido de casamento. Trad. Correia Alves. Os malefícios do tabaco. Trad. Luiz Francisco Rebello. 0 urso. Enc. Norberto Barroca. Prod. TEP.

Três irmãs. Trad. Mário Barradas. Enc. Mário Barradas. Prod. Centro Dramático de Évora (CENDREV).

A última dança. Coreografia: Elisa Worm. Prod. Ballet Contemporâneo do Norte [dança].

1999. Aniversário. Enc. Rui Sena. Prod. GICC - Teatro das Beiras.

The Cherry Orchard. Enc. Jonathan Weightman. Prod. Lisbon Players.

Retratos - 4 peças em 1 acto: Os malefícios do tabaco, Um pedido de casamento, 0 trágico à força e $\boldsymbol{O}$ aniversário do banco. Enc. João Bilou. Prod. SOIR.

Não está! Ou a saga do director geral: Os malefícios do tabaco. Enc. Luis Vicente. Prod. A Companhia de Teatro do Algarve (ACTA).

Los amores de Natália:Um pedido de casamento e $\boldsymbol{O}$ urso. Enc. Carlos Vides. Prod. Suripanta Teatro.

2000. 0 pedido de casamento. Enc. António Pereira. Prod. Grupo Cénico do Grupo de Instrução Popular da Amoreira.

Tio Vânia (Uncle Vanya, 1993). Autor: Howard Barker. Trad. Paulo Eduardo Carvalho. Enc. Rogério de Carvalho. Prod. As Boas Raparigas.

A gaivota. Trad. Regina Guimarães. Enc. Rui Madeira. Prod. Companhia de Teatro de Braga (CTB).

Tchekov Blues Bar - A casa das histórias [a partir de $O$ urso, Malefícios do tabaco e Um pedido de casamento]. Enc. Fernando Soares. Prod. Teatro do Carmo-Artes. 


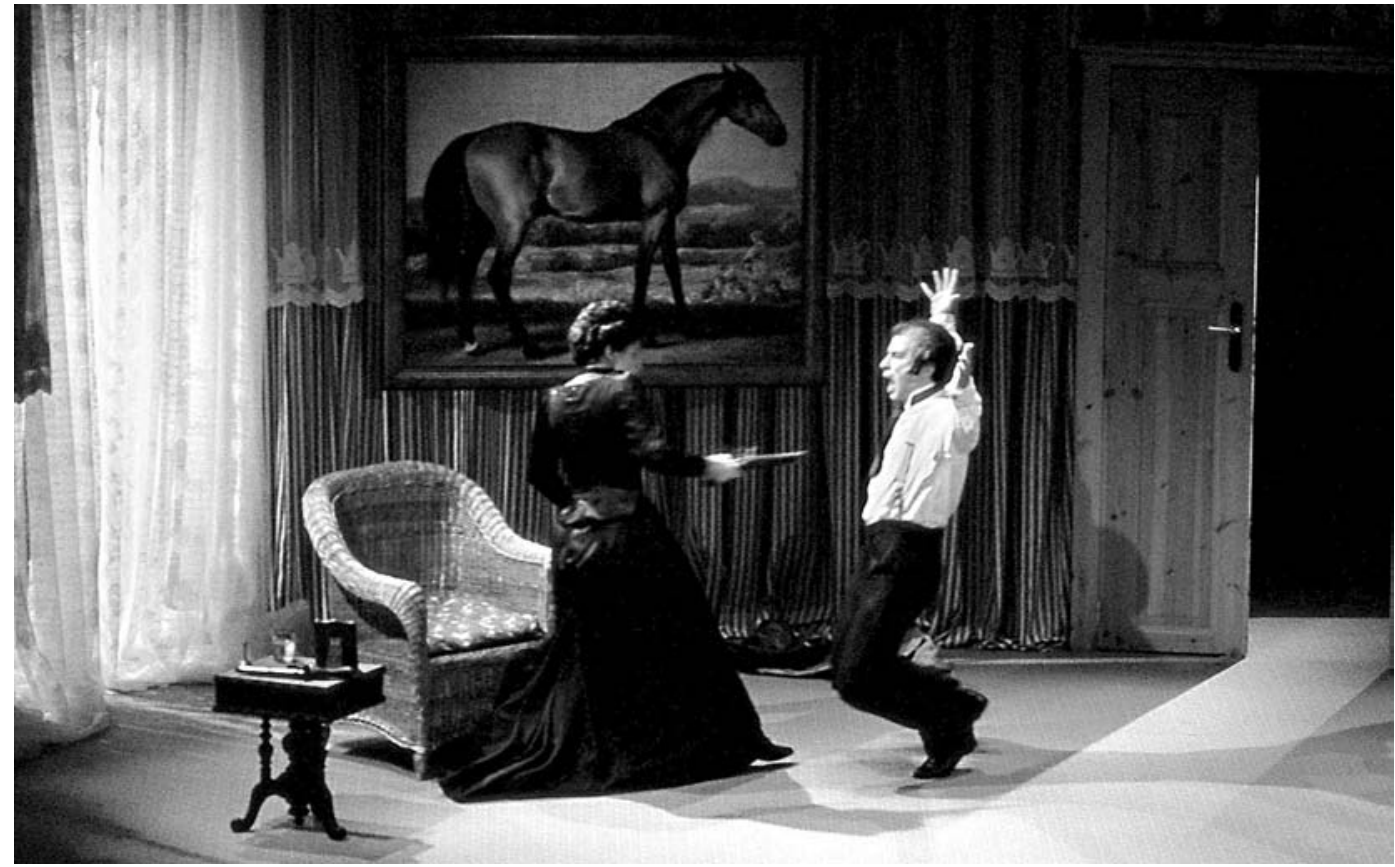

Malefícios do tabaco. Enc. José Caldeira.

Tio Vânia. Prod. Grupo de Teatro Miguel Torga Faculdade de Ciências Médicas da Universidade Nova de Lisboa.

Seis gaivotas: A gaivota. Trad. Fiama Hasse Pais Brandão. Concepção: Ana Vitorino, Carlos Costa, Catarina Martins e Pedro Carreira. Prod. Visões Úteis. 0 tio Vânia. Enc. Rogério de Carvalho. Prod. TEUC. Vidas medíocres: Um pedido de casamento, Os malefícios do tabaco e $O$ canto do cisne. Enc.

Júlio Correia. Prod. Companhia Municipal de Teatro de Sintra.

2001. ${ }^{5}$ Os malefícios do tabaco. Trad. Luiz Francisco

Rebello. Enc. Fátima Murta.

Pedido de casamento / 0 urso / Canto do cisne. Prod. Grupo Cénico da Sociedade Instrução Tavaredense.

A gaivota. Enc. Armando Caldas. Prod. Intervalo Grupo de Teatro.

O cerejal. Adap. e enc. António Rama. Prod. Bescénico.

Curtas [a partir de Tchekov]. Enc. César Alagoa. Prod. Teatro Experimental de Medicina (TEMA).

2002. Pano de muro: $O$ canto do cisne. Enc. Miguel Loureiro. Prod. Cão Solteiro.

O cerejal. Enc. Andreas Poppe. Prod. Baal 17 Companhia de Teatro na Educação do Baixo Alentejo.

Bater no fundo [integra Caixa preta de Lúcia Sigalho, a partir de A gaivota]. Dir. João Garcia Miguel. Prod. Olho - Associação Teatral / Sensurround.

Partitura inacabada [Platonov]. Trad. Filipe Guerra e Nina Guerra. Enc. Paulo Matos. Prod. Teatro da Trindade / Cine-Teatro Avenida.

$O$ albatroz [adaptação de A gaivota]. Autores: Mário Botequilha e Francisco Salgado. Enc. Francisco Salgado. Prod. Companhia do Chapitô. As três irmãs. Trad. Augusto Sobral, Carol Loff e Rui Mendes. Enc. Rogério de Carvalho. Prod. Companhia de Teatro de Almada.
Tchekovmédias: 0 urso, Um pedido de casamento Aniversário do banco. Enc. Jorge Gonçalves. Prod. Grupo de Artes e Comédias do Banco de Portugal. 0 aniversário no banco. Prod. Teatro de Ensaio do Barreiro.

Silence So Sexy [a partir de Três irmãs]. Dir. Francisco Camacho [dança].

Malefícios e outras virtudes do tabaco: Os malefícios do tabaco. Enc. João Paulo Janicas. Prod. Os Bonifrates.

2003. Ensaio (Ensaio, 2003). Autor: José Peixoto. Enc. José Peixoto. Prod. Teatro dos Aloés.

Quem tem medo de Anton Tchekov? [a partir de três textos de Tchekov] Enc. Bruno Bravo. Prod. Teatro Experimental do Funchal.

Os malefícios do tabaco. Enc. Florbela Oliveira.

Cerejal (materiais de trabalho). Concepção: Lúcia Sigalho. Prod. Sensurround.

Canto do cisne. Enc. Cão Solteiro - Miguel Loureiro. Prod. Cão Solteiro / Casa d'Os Dias da Água. Três irmãs - que importância é que isto tem? [a partir de Três irmãs]. Enc. Mónica Calle. Prod. Casa Conveniente.

Caixa preta - gaivota (Caixa preta, 2002). Autora: Lúcia Sigalho. Enc. Lúcia Sigalho. Prod. Sensurround. Uma peça mais tarde / 0 jogo de lalta (Afterplay, 2002 / The Yalta Game, 2001). Autor: Brian Friel. Trad. Paulo Eduardo Carvalho. Enc. Nuno Carinhas. Prod. Escola de Mulheres / Teatro Nacional S. João. As visitas: 0 urso e Um pedido de casamento. Enc. Paulo Madeira. Prod. Grupo Cénico Os 4 Ventos. Uma noite com Tchekov: Um pedido de casamento e $\boldsymbol{O}$ aniversário do banco. Enc. Graciano Simões. Prod. Teatro de Ensaio do Barreiro.

Malefícios do tabaco. Prod. Grupo Jograis e Trovadores.

Vânias [a partir de Tio Vânia]. Enc. António M. Rodrigues. Prod. Eclipse Arte.

2004. ${ }^{6}$ Duas comédias em um acto: 0 urso. Trad. Luiz Francisco Rebello. Um pedido de casamento. Trad.
Os Artistas Unidos

prepararam-se, neste ano, para estrear $\mathrm{Na}$ estrada com encenação de Jorge Silva Melo. A morte do actor Paulo Claro impediu a companhia de concretizar o projecto.

${ }^{6}$ Centenário da morte de Tchekov e da estreia de 0 Cerejal no Teatro de Arte de Moscovo. 


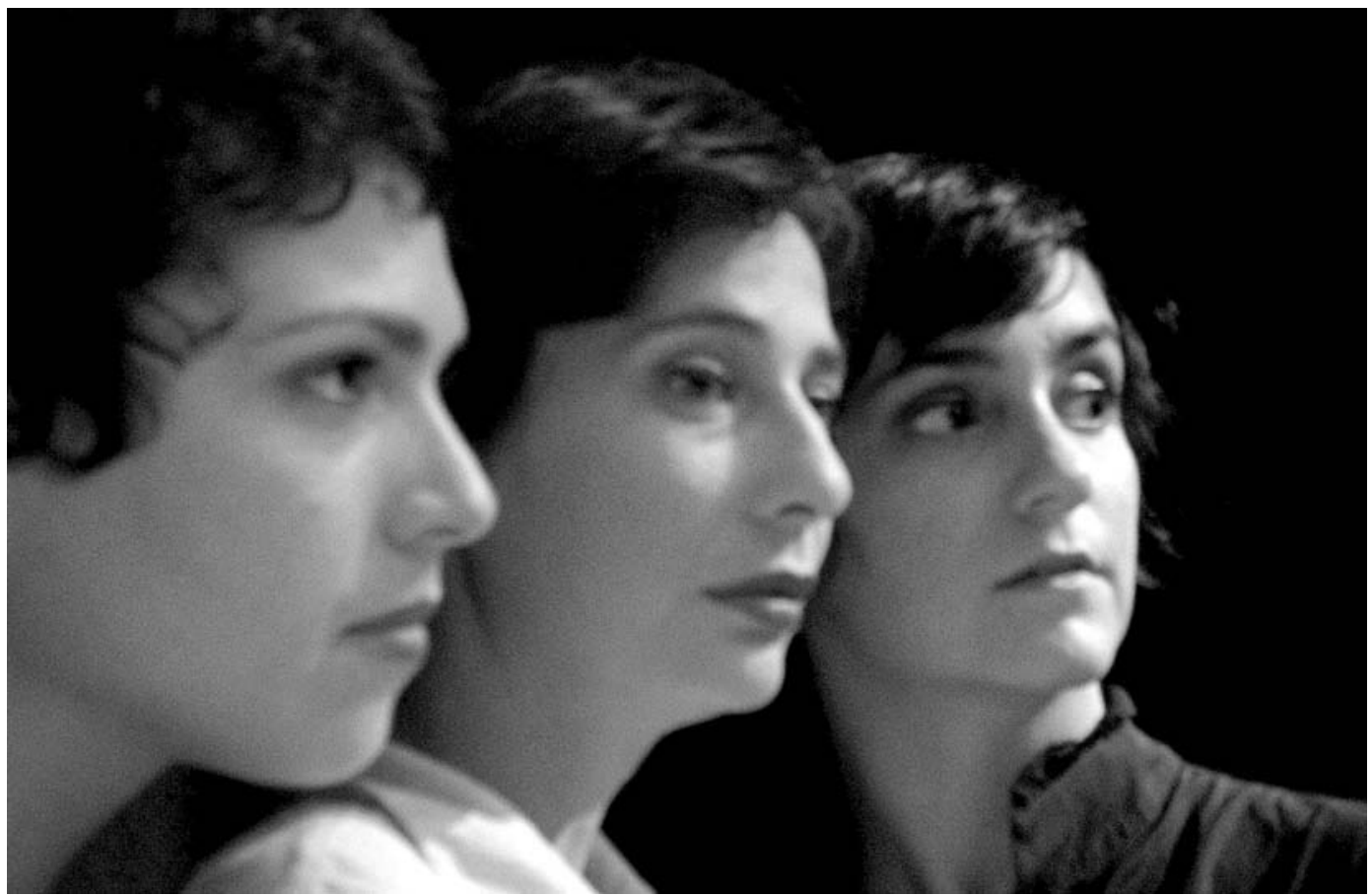

Uma peça mais tarde

+0 jogo de lalta,

enc. Nuno Carinhas, Escola de Mulheres/ TNSJ, 2003 (Sandra Faleiro e

João Lagarto), fot. João Tuna.
Correia Alves. Enc. Anabela Neves. Prod. Grupo de Teatro 0 Grito.

Humores [integra textos de Tchekov]. Dramaturgia: Fernando Rebelo. Enc. Fernando Rebelo. Prod. Oficina de Teatro de Almada.

Ser e não ser [integra excerto de 0 cerejal]. Enc. Maria do Céu Guerra. Prod. A Barraca.

Nina / Lá... é tudo melhor [a partir de Tchekov]. Autor: Rui Pina Coelho. Enc. Ana Falé e Rui Pina Coelho. Prod. Trimagisto - Cooperativa de Experimentação Teatral.

A boneca de Vassilissa. [integra contos de Tchekov]. Enc. Paulo Alexandre Lage. Prod. Companhia de Teatro Magia e Fantasia.

Você é um urso. Enc. João de Melo Alvim. Prod. Companhia de Teatro de Sintra.

Um pedido de casamento/ Os malefícios do tabaco. Prod. Grupo Desportivo de Lousa.

Tchekov: Os malefícios do tabaco, 0 urso. Prod. Teatro Independente de Loures. Enc. Carlos Paniágua. Um pedido de casamento/ $O$ urso. Enc. Ruy de Matos. Prod. Associação Coração Amarelo.

O cerejal. Trad. Carlos Porto. Versão: Rogério de Carvalho e Silvia Brito. Enc. Rogério de Carvalho. Prod. A Escola da Noite.

2005. A missão ou porque as raparigas continuam a querer ir para Moscovo [a partir de Três irmãs]. Enc. Mónica Calle. Prod. Casa Conveniente. Siráj [A gaivota]. Trad. Géza Morcsányi. Enc. Árpád Schilling. Prod. Teatro Krétakör.

\section{Referências bibliográficas}

FERREIRA, Armando (1923), "A directriz artistica do teatro moderno", De teatro, n. 5, Ano II, 3a série, Especial Nov./Dez.

LACERDA, Augusto de (1924), Teatro futuro:Visão de uma nova dramaturgia, Coimbra, Imprensa da Universidade.

NORONHA, Eduardo de (1909), Evolução do teatro: O drama através dos

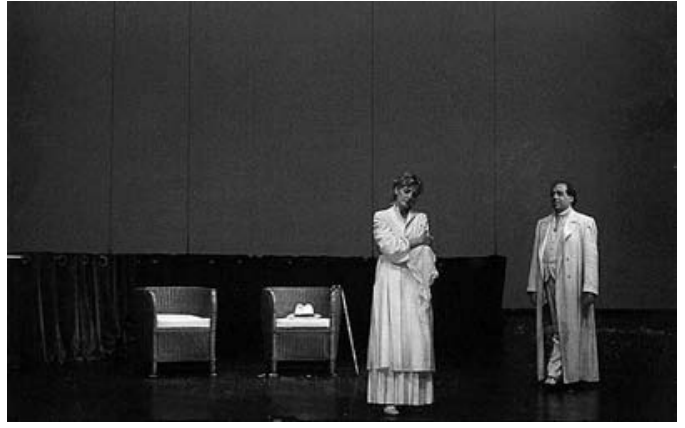

séculos. Compilação de vários estudos, Lisboa, Livraria Clássica Editora de A.M. Teixeira e C.

OLIVEIRA, Joaquim de (1950), 0 teatro novo: O Knock e o seu encenador. Subsidios para a técnica e história do teatro português, Lisboa, Livraria da Trindade.

PORTO, Carlos (1973), Em busca do teatro perdido, 2 vols., Lisboa, Plátano. - - (1997), O TEP e o teatro português: Histórias e imagens, Porto, Fundação Engenheiro António de Almeida.

RICARDO, Augusto (1934), Motivos de teatro, Prefácio de Araújo Pereira, Lisboa, Editor Nunes de Carvalho.

SCARLATTI, Eduardo (1927), Ideias de outros: Ensaios sobre literatura e estética teatral, seguidos de uma novela, Lisboa, Oficina Ottosgráfica. TCHEKOV, Anton (1960), Seis peças em um acto: Os maleficios do tabaco, O urso (versões de Luiz Francisco Rebello), Um pedido de casamento, 0 trágico à força, 0 aniversário do banco (versões de Correia Alves) e 0 canto do cisne (versão de Rui Sequeira), Porto, Divulgação (2a edição, Porto, Minotauro, 1965).

- - (1961), Duas peças em um acto: A boda e Na estrada real, trad. António Pinheiro Guimarães, Porto, Divulgação. 